\title{
Investigation on impact behavior of several structured design cushion spacers
}

\author{
W. Z. Zhong, G. Chen, Z. M. Hao, X. C. Huang, X. L. Wang, \\ S. Z. Li \& X. J. Liu \\ Institute of Systems Engineering, \\ China Academy of Engineering Physics, China
}

\begin{abstract}
The impact response of rubber and foam aluminum cushion spacers is simulated by ABAQUS finite element analysis software. It indicates that shape designed structures of rubber and foam aluminum buffer effectively in impact conditions. The rubber structure induces different deformation modes of the cushion spacer during the impact process and the deformation mode can be controlled. Elastic deformation of the rubber structure can accumulate energy. Rubber cushion endergonic and exergonic processes are reversible. It transforms kinetic energy into potential energy, prolongs loading time and releases the impact loading peak. Plastic deformation of foam aluminum can absorb energy and dissipation impacts kinetic energy. The process of aluminum dissipation energy is irreversible. Foam aluminum plastic dissipation energy is analyzed by a simplified mechanical theory. And corresponding impact experiments verify that the structured design rubber cushion spacer is valid.

Keywords: rubber, foam aluminum, impact cushion, energy transform, numerical simulation.
\end{abstract}

\section{Introduction}

Ideal cushion material is with wide range plastic plateau stress. Stress varies little in the plastic deformation process. Cushion material is classified into energy dissipation and energy storage. Energy dissipation material is used validly only once for an impact case, such as foam metal. Kinetic energy converts into elastic potential energy for energy storage material, such as rubber material and spring structure. And the cushion material can be reusable. The cushion material 
extends the duration of impact action and there is usually a smaller loading peak. Recently, investigations on material energy absorption have been done in the industrial and traffic fields. Cushion material and structure is applied widely in the product packaging buffer and highway guardrail.

Nowadays, a lot of study on cushion theory and energy absorption has been undertaken. For example, Alexander [1] analyzed the collapse behavior of thin cylindrical shells under axial loading and put forward a mean compression loading distribution expression. Li et al. [2] adopted the Euler-Lagrange variational principle to research the compression behavior of hollow cylinders composed of rubber sponge, and established nonlinear differential equations. Zhou et al. [3] designed a new metal rubber isolator. The experimental results demonstrated that the new metal rubber isolator is able to isolate vibration with a favorable vertical capacity and horizontal restorability. Guillow et al. [4] and ElHage et al. [5] studied the static axial compression mechanical behavior of thinwalled circular aluminum tubes. Yuan et al. [6] analyzed the inflation mechanism examined for a composite cylindrical tube composed of two incompressible rubber materials, where the inner surface of the tube was subjected to suddenly applied radial pressure. Hu et al. [7] adopted three methods to evaluate the cushioning properties and energy-absorption capability of rigid polyurethane foam. Zeng et al. [8] analyzed hard polyurethane foam plastics cushion properties in packaging engineering. Gu et al. [9] explored the dynamic features of four rubber complex materials composed of different admixtures when compact load exerted and analyzed their better energy absorbing effect and other researchers $[10,11]$ have undertaken studies on cushion material design and shock resistance.

In the paper, the impact response of a water gun with a rubber and foam aluminum cushion spacer is simulated by ABAQUS finite element analysis software. Deformation and failure modes are investigated. Foam aluminum plastic dissipation energy is analyzed by a simplified mechanical theory.

\section{Analysis model}

\subsection{Cushion model}

Compressible foam aluminum and hyperelastic rubber are taken as cushion materials in water gun equipment. As foam aluminum is with a wide ranging plastic compression plateau, kinetic energy is dissipated. For rubber, kinetic energy is transformed into potential energy. In order to investigate the buffer performance of different material structures, several foam aluminum and rubber cushion spacers are designed (shown in figure 1).

The foam aluminum structure is shown in figure 1 (a). The height is $150 \mathrm{~mm}$. Rubber cushions I, II and III are shown in figures 1(b), (c) and (d). The height of the rubber cushion spacers is $200 \mathrm{~mm}$ and there are some half round cutting grooves in the cushion spacers respectively. 


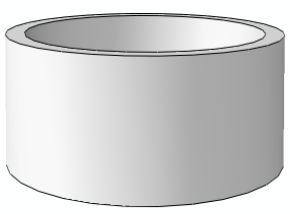

(a) foam aluminum

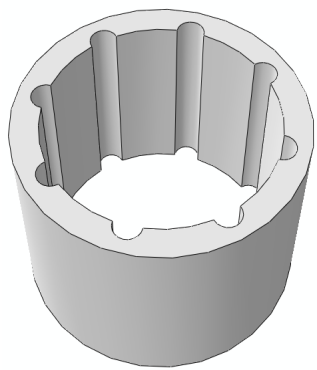

(c) rubber cushion II

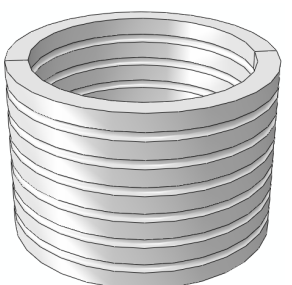

(b) rubber cushion I

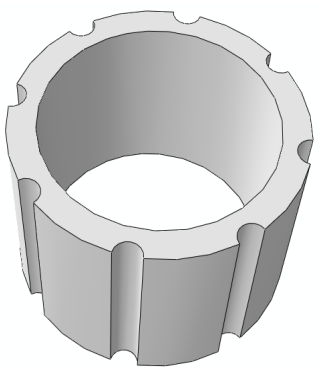

(d) rubber cushion III

Figure 1: Sketch of cushion spacers.

\subsection{Finite element model}

The finite element model of a water gun is established by ABAQUS software (shown in figure 2). There are approximately 57,000 hexahedral elements in the numerical model.

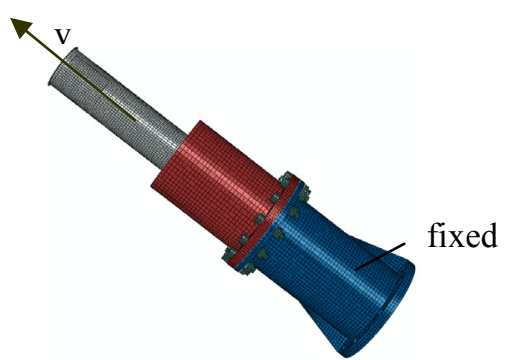

(a) whole model

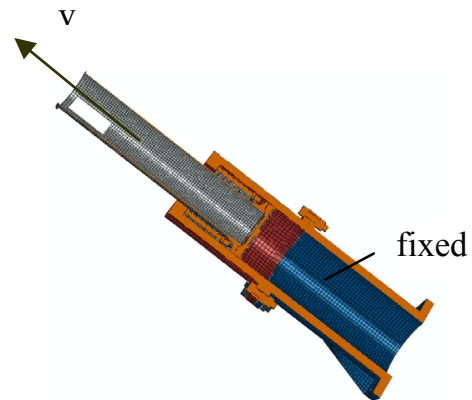

(b) half model

Figure 2: Finite element model. 


\subsection{Material mechanical behavior}

A compressible constitutive model is applied to describe the foam aluminum mechanical properties. For rubber, the Ogden model is adopted to describe strain potential energy. Compression stress versus strain curves of the two materials are shown in figure 3.

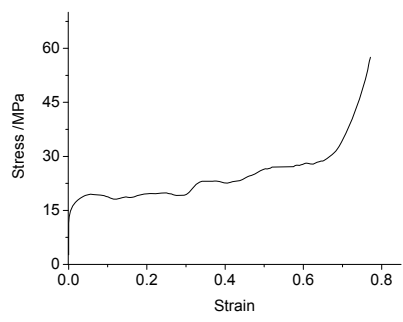

(a) foam aluminum

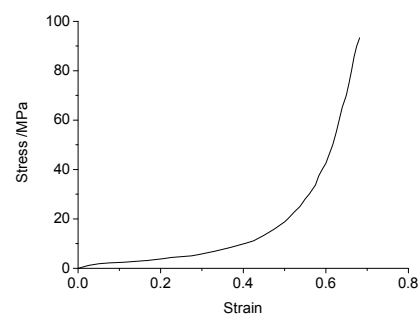

(b) rubber

Figure 3: Stress versus strain curves of foam aluminum and rubber.

\section{Finite element simulation}

The water gun impact response is simulated. The impact speed is $35 \mathrm{~m} / \mathrm{s}$. Numerical simulation results are shown in figure 4. It indicates that deformation of the water gun is elastic and the cushion spacers are effective under $35 \mathrm{~m} / \mathrm{s}$ impact condition. The foam aluminum structure becomes short during the impact
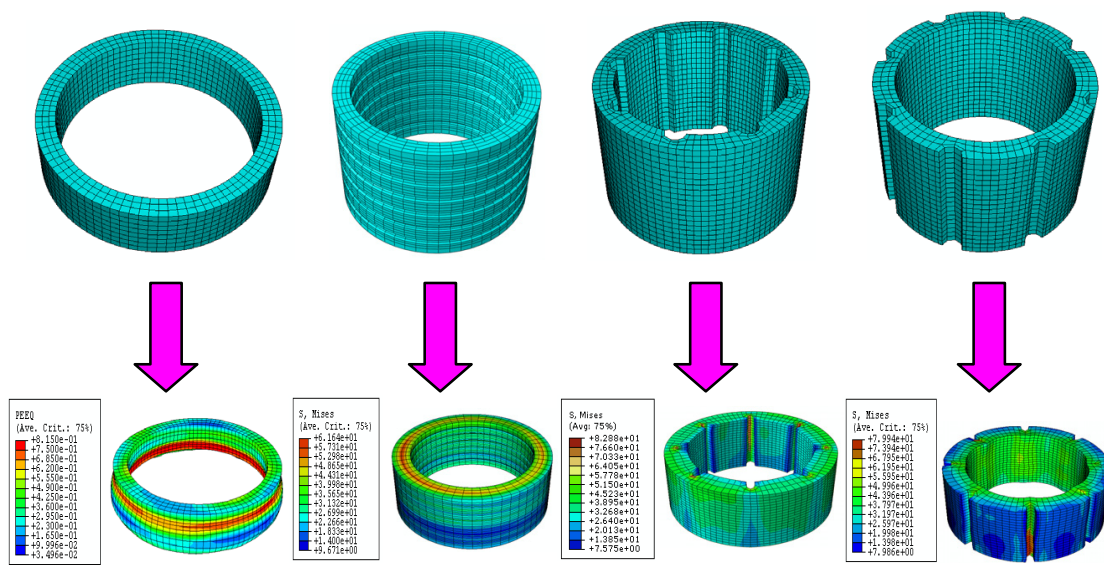
(c) rubber cushion II
(d) rubber cushion III
(a) foam aluminum
(b) rubber cushion I
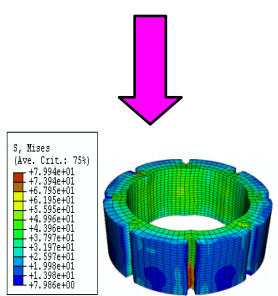

Figure 4: Deformation figures of cushion spacers. 
process. Radial and toroidal expansion does not appear (shown in figure 4(a)). Rubber cushion I occurs series folds and radial dimension is stable (shown in figure 4(b)). Rubber cushions II and III are extruded along the cutting grooves and the radial dimension changes little (shown in figures $4(\mathrm{c})$ and (d)).

\section{Discussion}

According to the simulation results, it indicates that a rubber cushion spacer can transform kinetic energy into potential energy, prolong loading time and weaken the impact loading peak. But plastic deformation of foam aluminum can absorb energy and dissipation structure impact kinetic energy.

For an aluminum spacer, the plateau stress can be taken as constant. Ignoring friction influence, the mass of water gun moving unit is given $M$. The initial speed is $v$. Plateau stress of aluminum is $\sigma_{y}$ and the cushion section area is $A$. The conservation relation of between kinetic energy and potential energy is expressed as follows

$$
E=\frac{1}{2} M v^{2}=\sigma_{y} A L \delta
$$

In equation (1), $L$ is the cushion spacer thickness. $\delta$ is the cushion spacer compression percent. Based on the principle of conservation of momentum and impact acting time $t$, equation (1) can be simplified as

$$
t=\frac{v}{a}=\frac{v M}{\sigma_{y} A}
$$

And cushion spacer deformation is expressed as equation (3)

$$
S=\frac{1}{2} a t^{2}=\frac{1}{2} \frac{M v^{2}}{\sigma_{y} A}
$$

In order to ensure that the water gun moving unit kinetic energy is dissipated completely by spacer plastic deformation, the minimum thickness is expressed as equation (4).

$$
L \Delta \geq \frac{1}{2} \frac{M v^{2}}{\sigma_{y} A}
$$

In equation (4), $\Delta$ is the compression percent when the spacer is compressed fully. Simplify equation (4) and a minimum thickness of spacer is gained.

$$
L \geq \frac{1}{2} \frac{M v^{2}}{\sigma_{y} A \Delta}
$$

\section{Conclusions}

Numerical simulation on cushion performance of four cushion spacers is carried out. It indicates that shape designed structures of rubber and foam aluminum are 
effective in impact cushion conditions. A rubber structure can induce different deformation modes of cushion spacer in impact state. Elastic deformation of the rubber structure can accumulate energy and release impact loading peak. Plastic deformation of foam aluminum can absorb energy and dissipation structure impacts kinetic energy and theory analysis on impact compression behaviour of aluminum is carried out. The process of aluminum dissipation energy is irreversible.

\section{Acknowledgements}

The authors gratefully acknowledge the funding by National Natural Science Foundation of China under the contract No. 11302211, 51174173 and National Basic Research Program of China (973 program) under the contract No. 2010CB832700.

\section{References}

[1] Alexander, J.M., An approximate analysis of the collapse of thin cylindrical shells under axial loading. Quarterly Journal of Mechanics and Applied Mathematics, 13, pp. 1-9, 1960.

[2] Li, S.J., Lin, G.W. \& Ma, D.W., The research on the axisymmetric plane strain problem of compressed hollow cylinder composed of elastomeric foam. Chinese Journal of Theoretical and Applied Mechanics, 42(3), pp. 579-582, 2010.

[3] Zhou, Y.G., Zhang, J. \& He, Y., Design and performance studying of vibration isolator using metal rubber. China Civil Engineering Journal, 43(s), pp. 352-355, 2010.

[4] Guillow, S.R., Lu, G. \& Grzebieta, R.H., Quasi static axial compression of thin-walled circular aluminum tubes. International Journal of Mechanical Sciences, 43(9), pp. 2103-2123, 2001.

[5] El-Hage, H., Mallick, P.K. \& Zamani, N., A numerical study on the quasistatic axial crush characteristics of square aluminum-composite hybrid tubes. Composite Structures, 73(4), pp. 505-514, 2006.

[6] Yuan, X.G., Zhang, W.Z., Zhang, H.W. \& Zhu Z.Y., Stability analysis of radial inflation of incompressible composite rubber tubes. Applied Mathematics and Mechanics, 32(3), pp. 286-292, 2011.

[7] Hu, S.S., Liu, J.F. \& Wang, W., Evaluation of cushioning properties and energy-absorption capability of rigid polyurethane foam. Explosion and Shock Waves, 18(1), pp. 42-47, 1998.

[8] Zeng, F., Pan, Y. \& Hu, S.S., Evaluation of Cushioning Properties and Energy absorption Capability of Foam Aluminium. Explosion and Shock Waves, 22(4), pp. 358-362, 2002.

[9] Gu, H.J., Zhao H.Z., Zhang, H.B. \& Zhang, W., Experimental study of the dynamic properties of high polymer complex material. Materials Science \& Engineering, 20(4), pp. 537-540, 2002. 
[10] Guo, T. \& Wang, Y.F., Energy absorbing structures imitating trabecular of beetle cuticles. Engineering Mechanics, 28(2), pp. 246-251, 256, 2011.

[11] Zu, X.D., Huang, Z.X. \& Gu, X.H., Experiment study of defense efficiency about rubber composite armor against shaped charge jet. Journal of Ballistics, 23(1), pp. 54-57, 2011.

[12] Du X.W, Song H.W., Impact dynamic and crashworthiness design of cylindrical structures, Beijing: Science Press, 2003. 